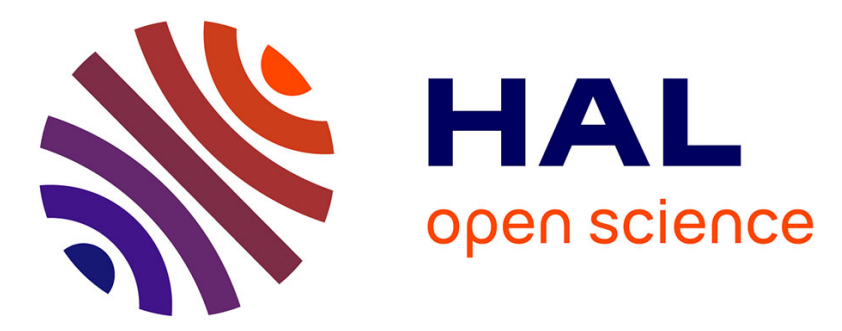

\title{
Etude d'un générateur électrochimique fonctionnant selon un cycle bitherme
}

B. Logé, J.F. Rialland, J. Robert

\section{To cite this version:}

B. Logé, J.F. Rialland, J. Robert. Etude d'un générateur électrochimique fonctionnant selon un cycle bitherme. Revue de Physique Appliquée, 1981, 16 (2), pp.37-47. 10.1051/rphysap:0198100160203700 . jpa-00244897

\section{HAL Id: jpa-00244897 https://hal.science/jpa-00244897}

Submitted on 1 Jan 1981

HAL is a multi-disciplinary open access archive for the deposit and dissemination of scientific research documents, whether they are published or not. The documents may come from teaching and research institutions in France or abroad, or from public or private research centers.
L'archive ouverte pluridisciplinaire HAL, est destinée au dépôt et à la diffusion de documents scientifiques de niveau recherche, publiés ou non, émanant des établissements d'enseignement et de recherche français ou étrangers, des laboratoires publics ou privés. 


\title{
Etude d'un générateur électrochimique fonctionnant selon un cycle bitherme
}

\author{
B. Logé, J. F. Rialland et J. Robert \\ LGEP-ESE, Plateau du Moulon, 91190 Gif-sur-Yvette, France \\ (Reçu le 6 mai 1980, réviséle 27 octobre 1980, accepté le 7 novembre 1980)
}

\begin{abstract}
Résumé. - Le présent travail est relatif à la réalisation et à l'expérimentation d'un générateur électrochimique fonctionnant selon un cycle bitherme. Deux compartiments constituant respectivement la source chaude et la source froide sont séparés par une paroi d'alumine $\beta$. Ces deux compartiments contiennent du sodium. Il en résulte un gradient de potentiel chimique entre les fractions de métal alcalin respectivement dans les conditions thermodynamiques de la source chaude et celles de la source froide. De l'énergie électrique peut être récupérée en transférant le sodium de la première à la seconde à travers l'alumine $\beta$. La f.é.m. de ce générateur, mesurée expérimentalement est comparée à sa valeur théorique. L'analyse des essais en charge conduit à penser que le transfert des charges de l'alumine $\beta$ au collecteur du côté de la source froide limite principalement les performances du dispositif. Ceci résulte du fait que le sodium métallique est alors en phase dispersée. Enfin, le rendement théorique de conversion est évalué pour diverses températures de source chaude, la source froide étant à la température de fusion du sodium.
\end{abstract}

\begin{abstract}
The present work is concerned with the achievement and testing of an electrochemical generator working in a bithermal cycle : the hot source and the cold source are held in twin compartments with between a dividing wall of $\beta$ alumina ; these two compartments contain a certain amount of sodium. This leads to a chemical potential gradient between the parts of alkaline metal which are under the respective thermodynamical conditions of the hot and cold source. Some electrical energy can be recovered by driving through the $\beta$ alumina sheet the sodium from the first compartment to the other. The measured value of the e.m.f. of this generator is compared to the theoretical result. By analysing the loading tests we are led to consider that the charge transfer from the $\beta$ alumina sheet to the collector facing the cold source is the main cause that accounts for the limited efficiency of this device, and this is due to the fact that the metallic sodium is then in a scattered phase. One last thing : the theoretical conversion efficiency is evaluated for various temperatures of the hot source while the cold source remains at the sodium melting temperature.
\end{abstract}

1. Introduction. - Le présent travail concerne l'étude et la réalisation d'un générateur permettant la conversion directe d'énergie thermique en énergie électrique. Le principe retenu est celui d'un générateur électrochimique fonctionnant selon un cycle bitherme.

Considérons un électrolyte divisant une cellule électrochimique en deux compartiments contenant un métal placé respectivement dans les conditions thermodynamiques de la source chaude et de la source froide. Il existe de part et d'autre de l'électrolyte une différence des activités des atomes métalliques se traduisant par l'apparition d'une force électromotrice. Le branchement d'un circuit électrique aux bornes de la cellule s'accompagne d'un transfert de métal de la source chaude à la source froide selon le schéma suivant :

- oxydation des atomes métalliques $M$ à l'interface de l'électrolyte en contact thermique avec la source chaude,
- migration des ions $\mathrm{M}^{+}$à travers l'électrolyte, - réduction des ions en atomes $M$ au niveau de l'interface en regard de la source froide.

La force électromotrice recueillie aux bornes d'un tel générateur est d'autant plus importante que l'écart de température est grand, et les performances d'autant meilleures que les cinétiques associées à chaque processus sont rapides, donc les températures élevées. Enfin, l'électrolyte doit servir de séparateur mécanique entre les deux compartiments. Ces impératifs conduisent à envisager l'utilisation d'un électrolyte solide.

Le principe d'une telle conversion est général. Cependant, la difficulté de trouver un électrolyte solide adapté limite pratiquement son développement aux métaux alcalins et plus particulièrement au sodium. Nous avons donc réalisé un dispositif expérimental dans lequel le métal est du sodium et l'électrolyte solide de l'alumine $\beta$. Parmi les conducteurs 
ioniques actuellement utilisés, l'alumine $\beta$ est à notre connaissance celui dont les propriétés sont les mieux définies et dont le développement à l'échelle industrielle est envisagé à court terme [1,2]. Les températures de fusion $\left(97,5^{\circ} \mathrm{C}\right)$ et d'ébullition $\left(892^{\circ} \mathrm{C}\right)$ du sodium permettent son utilisation à l'état liquide ou sous forme de vapeur sans difficultés technologiques excessives. Enfin, le caractère électropositif du sodium favorise la cinétique des réactions.

2. Description du générateur. - 2.1 PRINCIPE. Le convertisseur est essentiellement constitué d'un électrolyte solide divisant la cellule électrochimique en deux compartiments (Fig. 1). Le premier contient du sodium liquide, en équilibre avec sa vapeur, placé dans les conditions thermodynamiques de la source chaude $\left(p_{1}, T_{1}\right)$. L'interface supérieure de l'alumine $\beta$ est en contact thermique direct avec la source chaude. Afin d'éviter un court-circuit thermodynamique, il n'en est pas de même de la face inférieure de l'électrolyte vis-à-vis de la source froide. Celle-ci est à une distance telle que les transferts de chaleur par conduction peuvent être négligés. Il existe donc un gradient de température le long de ce compartiment. Au niveau de la source froide, le sodium se trouve dans les conditions thermodynamiques $\left(p_{2}, T_{2}\right)$, où $p_{2}$ représente la pression de vapeur saturante de sodium condensé à la température $T_{2}$. Entre la source froide et l'électrolyte, le sodium est à l'état de vapeur à une température $T$ intermédiaire entre $T_{1}$ et $T_{2}$ et sous une pression qui, en première analyse, peut être identifiée à $p_{2}$.

De part et d'autre de l'électrolyte, les équilibres suivants s'établissent :

$$
\begin{aligned}
\mathrm{Na}(\text { vap. })_{p_{1}} & \rightleftharpoons \mathrm{Na}^{+}+\mathrm{e} \\
\mathrm{Na}^{+}+\mathrm{e} & \rightleftharpoons \mathrm{Na}(\text { vap.) })_{p_{2}} .
\end{aligned}
$$

Si on admet que les équilibres s'effectuent à la température de la source chaude $T_{1}$, la force électromotrice est donnée en première approximation par la relation [3] :

$$
E=\frac{R T_{1}}{F} \ln \frac{p_{1}}{p_{2}}
$$

La conversion d'énergie thermique en énergie électrique s'accompagne de processus suivants :

$$
\begin{array}{ll}
(\mathrm{Na})_{p_{1}, T_{1}} \rightarrow \mathrm{Na}^{+}+\mathrm{e} & \begin{array}{l}
\text { Oxydation des atomes de so- } \\
\text { dium côté source chaude. } \\
\mathrm{Na}^{+} \rightarrow \mathrm{Na}^{+}
\end{array} \\
\mathrm{Na}^{+}+\mathrm{e} \rightarrow(\mathrm{Na})_{p_{2}, T_{1}} & \begin{array}{l}
\text { Migration des ions à travers } \\
\text { l'éctrolyte. }
\end{array} \\
& \begin{array}{l}
\text { Réduction des ions côté source } \\
\text { froide. }
\end{array} \\
(\mathrm{Na})_{p_{2}, T_{1}} \rightarrow(\mathrm{Na})_{p_{2}, T_{2}} & \begin{array}{l}
\text { Diffusion des atomes formés } \\
\text { jusqu’à la source froide et } \\
\text { condensation. }
\end{array}
\end{array}
$$

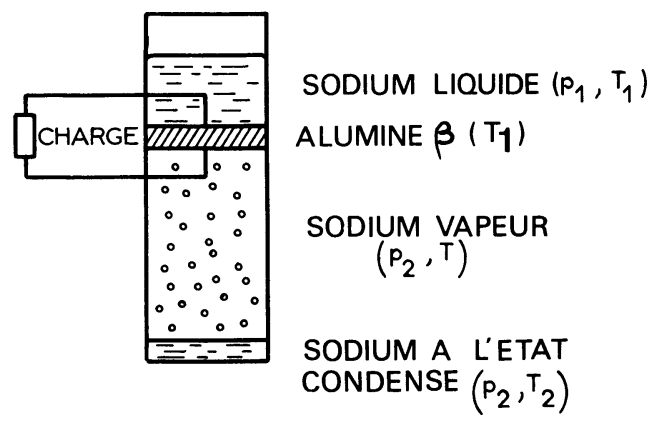

Fig. 1. - Principe de conversion.

[Conversion principle.]

La réalisation de contacts électriques assurant le transfert des électrons des zones réactionnelles au circuit extérieur soulève des difficultés surtout au niveau de l'interface électrolyte-sodium située en regard de la source froide. En effet, la phase métallique étant alors peu dense, la collection de courant implique l'existence d'une électrode en liaison étroite avec l'électrolyte et qui permette la diffusion des atomes vers la source froide.

Le gradient de pression nécessaire à l'apparition d'une force électromotrice est liée à la présence d'une source chaude et d'une source froide. Il serait possible de l'engendrer à l'aide de dispositifs mécaniques (compresseur et pompe à vide). Le générateur, alors monotherme, convertirait l'énergie mécanique en énergie électrique, mais ne présenterait de toute évidence aucun intérêt.

2.2 Dispositif EXPÉRIMENTAL. - L'alumine $\beta$ nous est fournie par le laboratoire de recherches de la Compagnie Générale d'Electricité $\left({ }^{1}\right)$. Ce dernier a en effet mis au point un procédé de fabrication [4], et de traitement du matériau [5], en vue de l'expérimentation d'accumulateurs sodium-soufre. Les échantillons se présentent sous forme d'un cylindre fermé à une extrémité par une calotte hémisphérique (doigt de gant). Ils sont obtenus par dépôt électrophorétique d'alumine $\beta$ sur un mandrin métallique, puis frittage à une température comprise entre 1800 et $1850^{\circ} \mathrm{C}$. Cette géométrie a conditionné la structure $\mathrm{du}$ convertisseur. L'alumine utilisée a une formule intermédiaire entre $10 \mathrm{Al}_{2} \mathrm{O}_{3}, \mathrm{Na}_{2} \mathrm{O}$ et $9 \mathrm{Al}_{2} \mathrm{O}_{3}, \mathrm{Na}_{2} \mathrm{O}$.

La cellule est représentée sur la figure 2. Le compartiment supérieur est constitué d'un tube d'alumine $\beta$ raccordé à un tube de verre. Une électrode en molybdène, scellée sur celui-ci, permet la collection du courant. Avant scellement du réservoir inférieur réalisé en Pyrex, la face externe du tube d'alumine $\beta$ est munie d'un collecteur. Celui-ci est constitué d'un fil de platine de $0,2 \mathrm{~mm}$ de diamètre enroulé de manière à former 29 spires non jointives et fixé par deux

\footnotetext{
$\left.{ }^{1}\right)$ Que nous tenons à remercier.
} 


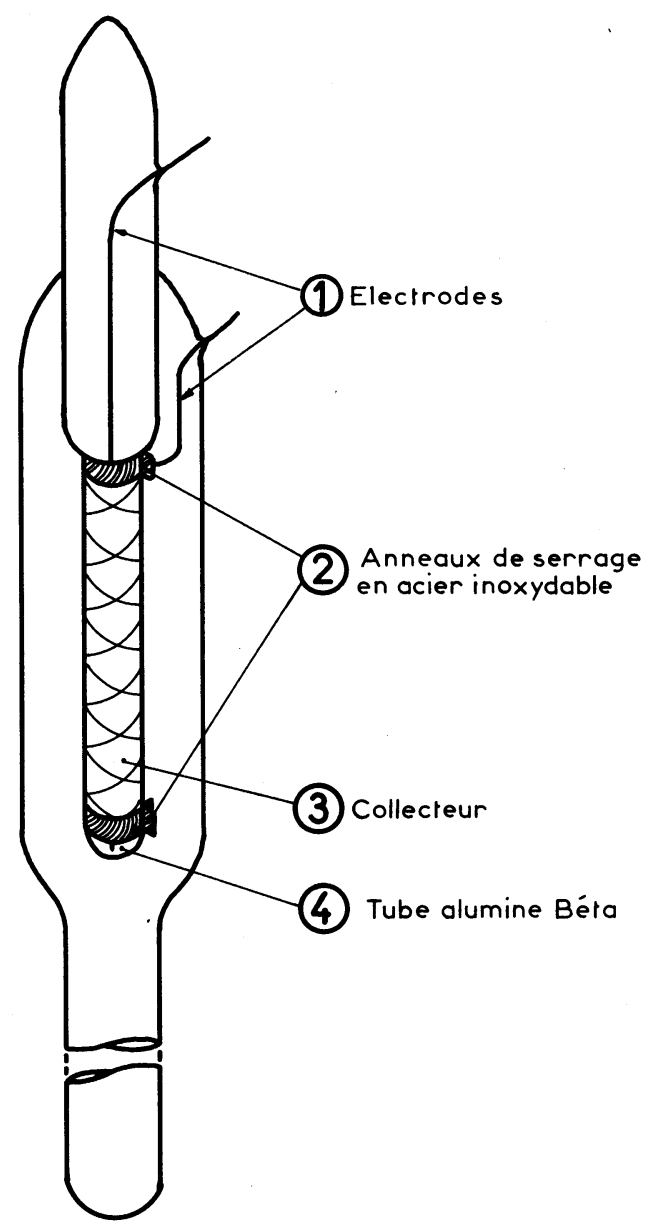

Fig. 2. - Cellule expérimentale.

[Experimental cell.]

anneaux de serrage en acier inoxydable. L'anneau supérieur est relié à une électrode en molybdène scellée dans le tube enveloppe.

Le sodium est introduit par soutirage sous forme liquide à l'intérieur du tube d'alumine $\beta$. Dans ce but, le compartiment supérieur est raccordé à un réservoir muni de picots de verre et d'un filtre de verre fritté dont le diamètre moyen des pores est d'environ $200 \mu \mathrm{m}$; ce réservoir est lui-même relié à un bâti de pompage (Fig. 3). L'ensemble de ce dispositif permet l'obtention dans. la cellule électrochimique d'un métal exempt d'impuretés (oxydes en particulier). $\mathrm{Au}$ terme de cette opération, la cellule et le réservoir sont séparés par scellement au niveau du queusot ménagé à cet effet. Le générateur est alors conforme à la figure 2 .

Afin d'éviter les risques d'oxydation spontanée du sodium, il faut limiter au mieux la présence d'oxygène dans l'ensemble du dispositif. Avant chargement de la cellule en sodium, on réalise un vide secondaire (environ $10^{-6}$ torr) dans chaque partie du montage, remplie préalablement d'argon U. Durant cette opération, la cellule est étuvée à $250^{\circ} \mathrm{C}$ afin d'éliminer les molécules d'eau adsorbées sur les parois. Cette

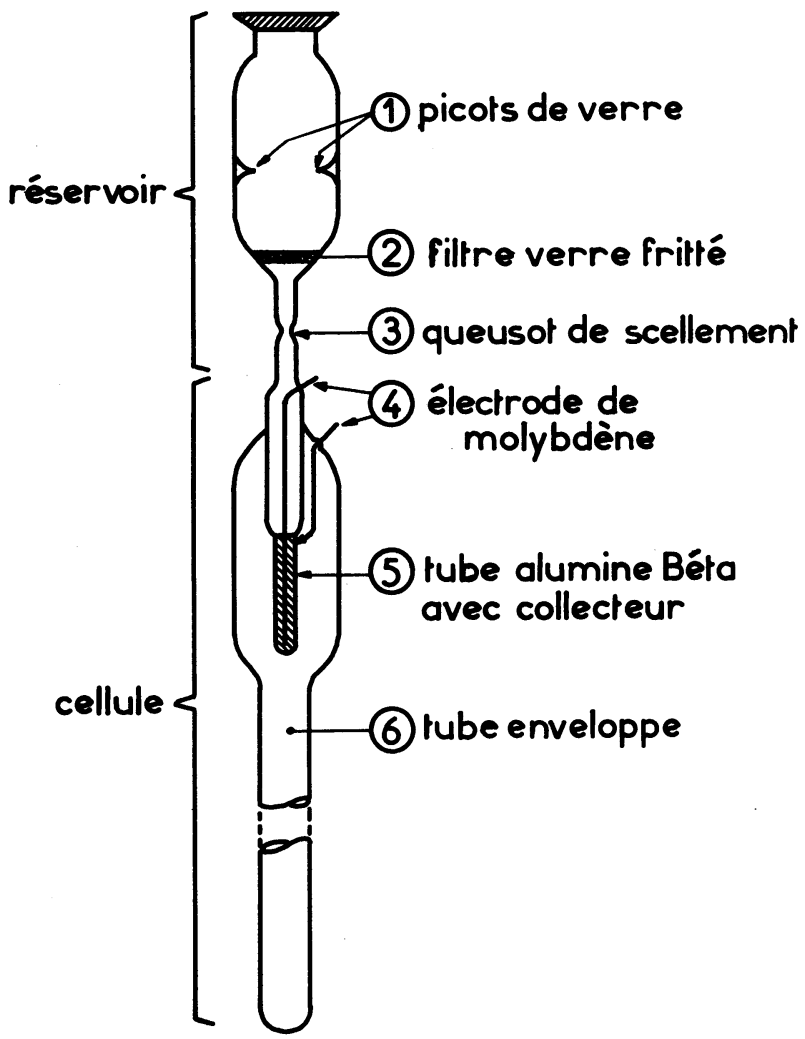

Fig. 3. - Dispositif de remplissage de la cellule.

[Cell filling device.]

procédure a permis d'effectuer l'ensemble des essais sans altération visible du métal alcalin.

Les dimensions des divers éléments constituant le générateur (Fig. 2) sont les suivantes :

- la longueur du tube d'alumine $\beta$ est $60 \mathrm{~mm}$, son diamètre extérieur $9 \mathrm{~mm}$, son épaisseur $1 \mathrm{~mm}$,

- le diamètre du tube enveloppe est dans la zone la plus large $45 \mathrm{~mm}$ et dans la zone la plus étroite $20 \mathrm{~mm}$; la longueur de l'enveloppe est $900 \mathrm{~mm}$. Cette valeur résulte de la nécessité décrite ci-dessus d'éloigner la source froide de l'électrolyte,

- l'épaisseur des parois des deux compartiments de $1 \mathrm{~mm}$ autorise l'utilisation de la cellule jusqu'à $500^{\circ} \mathrm{C}$ sans altération mécanique.

Le chauffage de la cellule est assuré à l'aide d'un four tubulaire d'une puissance nominale de $3 \mathrm{~kW}$ dont la tension d'alimentation est réglable. Ce four a une constante de temps thermique suffisante pour que, durant chaque mesure, les températures aux différents niveaux du générateur soient stables sans qu'il soit nécessaire de faire appel à un système de régulation. L'intérieur du four est chemisé à l'aide d'un tube de quartz, d'épaisseur $10 \mathrm{~mm}$, fermé à son extrémité inférieure. Ce tube assure l'isolation entre l'enroulement chauffant et la cellule en cas d'incident. Un couvercle de laiton muni d'un joint torique en viton permet de maintenir dans le tube de quartz une atmosphère d'argon réduisant les risques liés à la mani- 
pulation de sodium. Le schéma du montage est représenté sur la figure 4 .

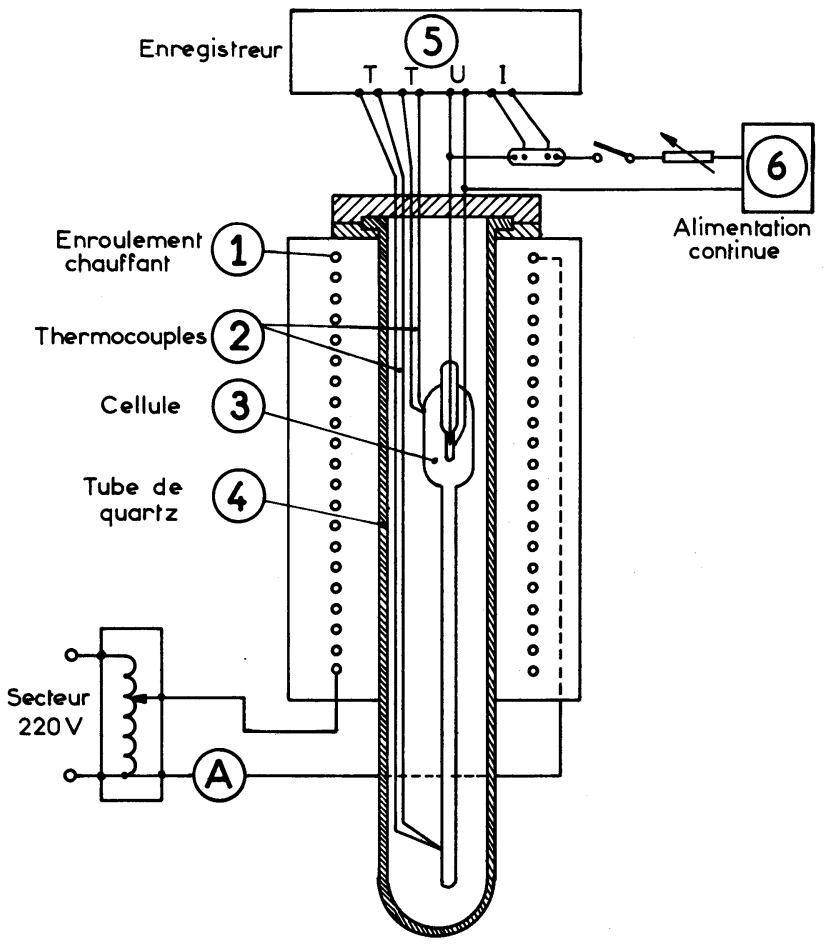

Fig. 4. - Schéma électrique du dispositif expérimental.

[Electrical diagram of the experimental device.]

Trois tiges filetées, fixées à ce couvercle assurent la rigidité d'une enceinte métallique cylindrique dans laquelle est placée la partie supérieure de la cellule (Fig. 5). La position de cette dernière par rapport au four peut ainsi être ajustée. L'insertion de fiches d'isolant thermique (fiber frax $\mathrm{H}$ ) permet de réduire les pertes par convection ainsi que le gradient thermique au niveau de l'alumine. Le fond de l'enceinte est muni d'un orifice permettant le passage de la partie tubulaire de la cellule dont l'extrémité inférieure constitue la source froide du générateur.

Les courbes représentées sur la figure 6 rendent compte des profils thermiques observés expérimentalement le long de la cellule pour plusieurs températures de source chaude. Il apparaît donc un gradient thermique au niveau de l'enceinte contenant le tube d'alumine $\beta$. Celui-ci est cependant faible devant l'écart de température entre le point le plus chaud et le point le plus froid de la cellule. La température de la source chaude est repérée par un thermocouple placé à mi-hauteur du tube d'alumine $\beta$ (niveau 1) et correspond à une valeur moyenne. Au cours des essais du générateur, seuls les thermocouples situés aux niveaux de la source chaude et de la source froide sont conservés. Les mesures de température sont effectuées avec une précision d'environ $3^{\circ} \mathrm{C}$.

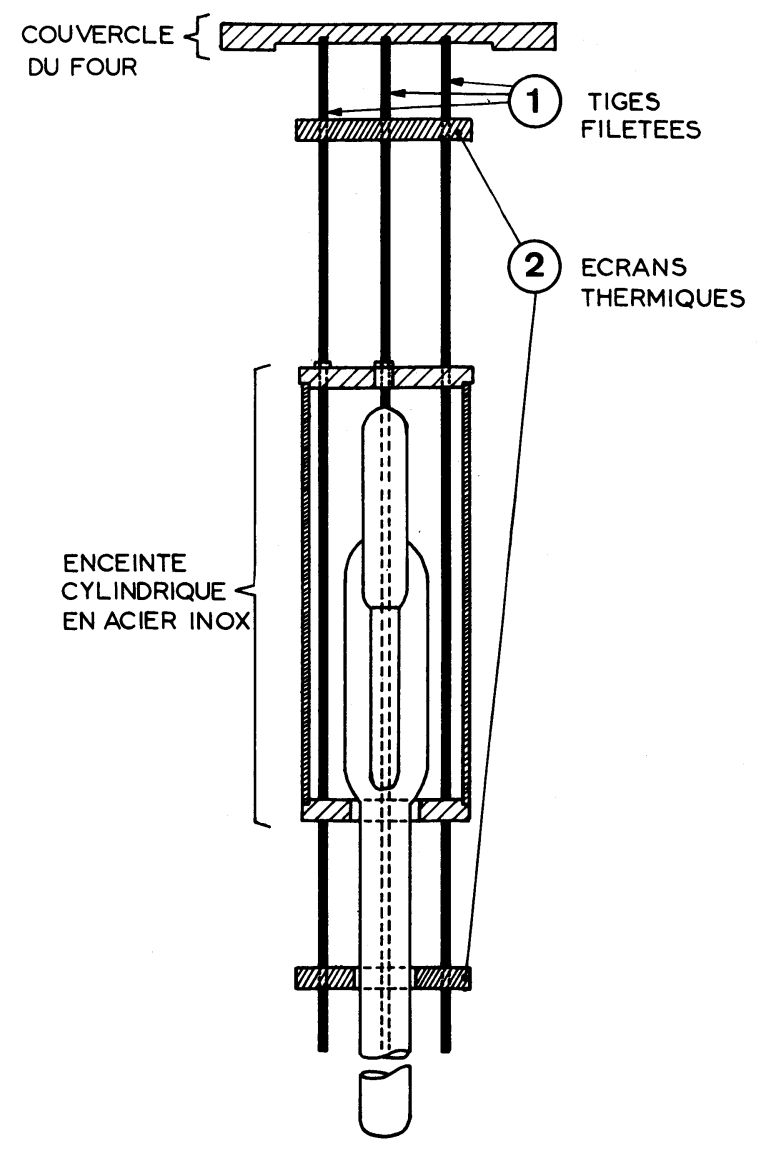

Fig. 5. - Dispositif de positionnement de la cellule dans un four vertical.

[Setting device for a cell placed in a vertical furnace.]

3. Etude du générateur. - 3.1 ETUDE DE LA FORCE ÉLECTROMOTRICE. - Les mesures de la f.é.m. du générateur ont été effectuées pour des températures de source chaude comprises entre $270^{\circ}$ et $530^{\circ} \mathrm{C}$, après stabilisation thermique du four. Dans ce domaine de température, on peut admettre que la température de la source froide reste constante (Fig. 6).

Les variations de la f.é.m. en fonction de la température sont précisées sur la figure 7 . Les résultats obtenus par Kummer et Weber [6], sur une cellule de conception analogue sont également indiqués; ceux-ci sont en relativement bon accord avec nos mesures. On constate que dans le domaine de température exploré, la loi de variation est linéaire.

Lors d'essais en charge (voir ci-dessus) réalisés à $T_{1}=270^{\circ} \mathrm{C}$ et $463^{\circ} \mathrm{C}$, les variations de la tension $V$ aux bornes de la cellule sont relevées en fonction du courant $I$ dans le cas d'un fonctionnement en générateur $(I>0)$ et en récepteur $(I<0)$. L'intersection de chaque courbe avec l'axe des ordonnées $(I=0)$ fournit la f.é.m. à la température considérée. Les valeurs obtenues sont comparables à celles mesurées lors des essais à vide.

La force électromotrice du générateur est reliée 


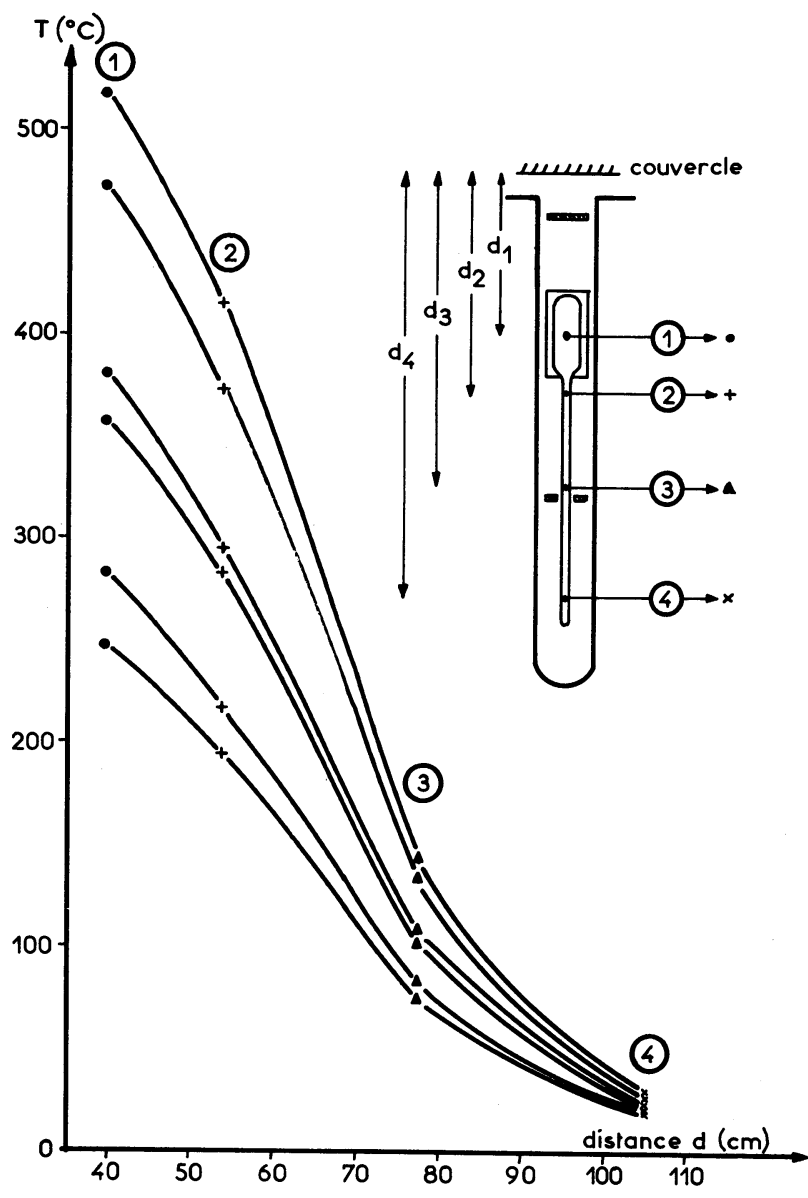

Fig. 6. - Gradient thermique au sein de la cellule.

[Thermal gradient within the cell.]

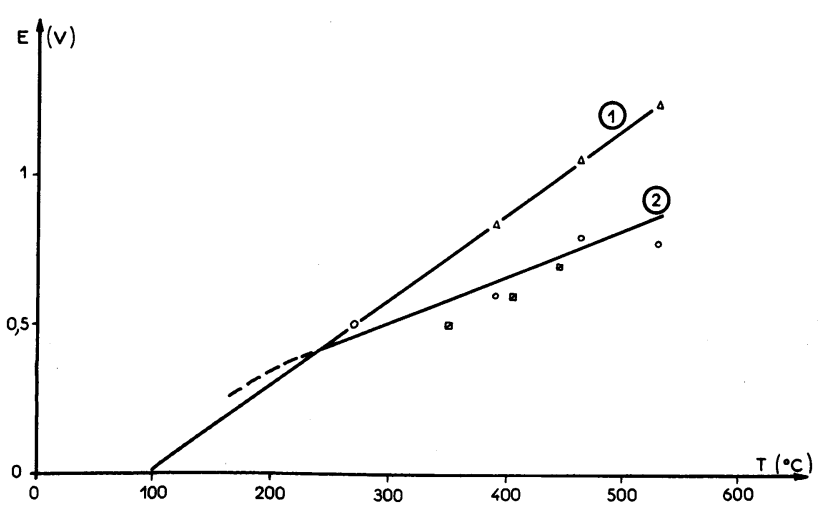

Fig. 7. - f.é.m. à vide : 1 théorique; 2 mesurée; $\square$ mesurée selon J. T. Kummer [15].

[E.m.f. when load is off : 1 theoretical ; 2 measured; $\square$ measured according to J. T. Kummer [15].]

aux activités respectives du sodium situé sur les sites réactionnels et donc à la surface de chaque collecteur. En supposant que les équilibres d'oxydo-réduction s'effectuent à la température $T_{1}$, la f.é.m. théorique est donnée en première approximation par l'équation (1), sous réserve que l'on admette que le sodium situé sur chaque collecteur est en équilibre avec la vapeur environnante. Les pressions $p_{1}$ et $p_{2}$ ne sont respectivement fonctions que des températures de source chaude et de source froide $T_{1}$ et $T_{2}$. La source chaude est située dans une zone du four où le gradient thermique est faible, et $T_{1}$ donc $p_{1}$, sont deux paramètres réglables. La température $T_{2}$ est connue avec une plus grande incertitude. On serait tenté d'admettre que la température de la source froide est celle du point le plus froid du montage, $30^{\circ} \mathrm{C}$ environ, à l'extrémité inférieure du générateur (Fig. 6). En fait, l'examen des cellules après fonctionnement montre que la majeure partie du sodium ayant participé à la réaction électrochimique s'est condensée près de la zone inférieure du four. La température correspondante est de l'ordre de $100^{\circ} \mathrm{C}$ et ne dépend pratiquement pas de la température $T_{1}$ (tout au moins dans la gamme explorée). Les processus de condensation en présence d'un gradient thermique ne sont pas simples. Si l'équilibre correspond à un état où toute la phase condensée est située au point le plus froid, le temps nécessaire à son obtention peut être suffisamment long (par rapport à la durée de l'expérience) pour qu'un état différent apparaisse comme l'état final. Nous avons admis que $p_{2}$ est la pression imposée par l'équilibre entre la vapeur et la phase condensée à la température de $100^{\circ} \mathrm{C}$.

La variation de la pression de vapeur saturante du sodium en fonction de la température est précisée sur la figure 8. Il apparaît que la pression de la vapeur saturante en équilibre avec le liquide en surfusion est comparable au voisinage du point de fusion à celle de la vapeur en équilibre avec le solide. Dès lors $p_{2}$ peut être déterminé sans qu'il soit nécessaire de préciser si la phase condensée à la source froide est à l'état liquide ou solide.

Dans ces conditions, la f.é.m. théorique est reliée à $T_{1}$ et $T_{2}$ par :

$$
E=1,09 \frac{T_{1}-T_{2}}{T_{2}}
$$

où $T_{1}$ et $T_{2}$ sont exprimés en $K$ et $E$ en $\mathrm{V}$. Le graphe $E\left(T_{1}\right)$ est tracé sur la figure 7; comme il a été dit la température $T_{2}$ est choisie égale à $373 \mathrm{~K}$ quelle que soit la valeur de $T_{1}$. On observe que les valeurs calculées sont supérieures aux valeurs expérimentales, l'écart augmentant avec la température. Cela peut être dû au fait que l'identification de l'activité à la pression de vapeur est d'autant moins justifiée que la pression est élevée et donc que la température est importante. De plus, $T_{2}$ n'est pas constante en toute rigueur.

3.2 ETUde du GÉNÉRATEUR EN CHARGe. - Le montage est schématisé sur la figure 9. Une alimentation, stabilisée en tension, branchée en série avec une résistance variable entre $1 \mathrm{k} \Omega$ et $1 \mathrm{M} \Omega$ permet d'imposer à travers la cellule un courant ajustable entre quelques $\mu \mathrm{A}$ et plusieurs dizaines de $\mathrm{mA}$. Un inverseur 


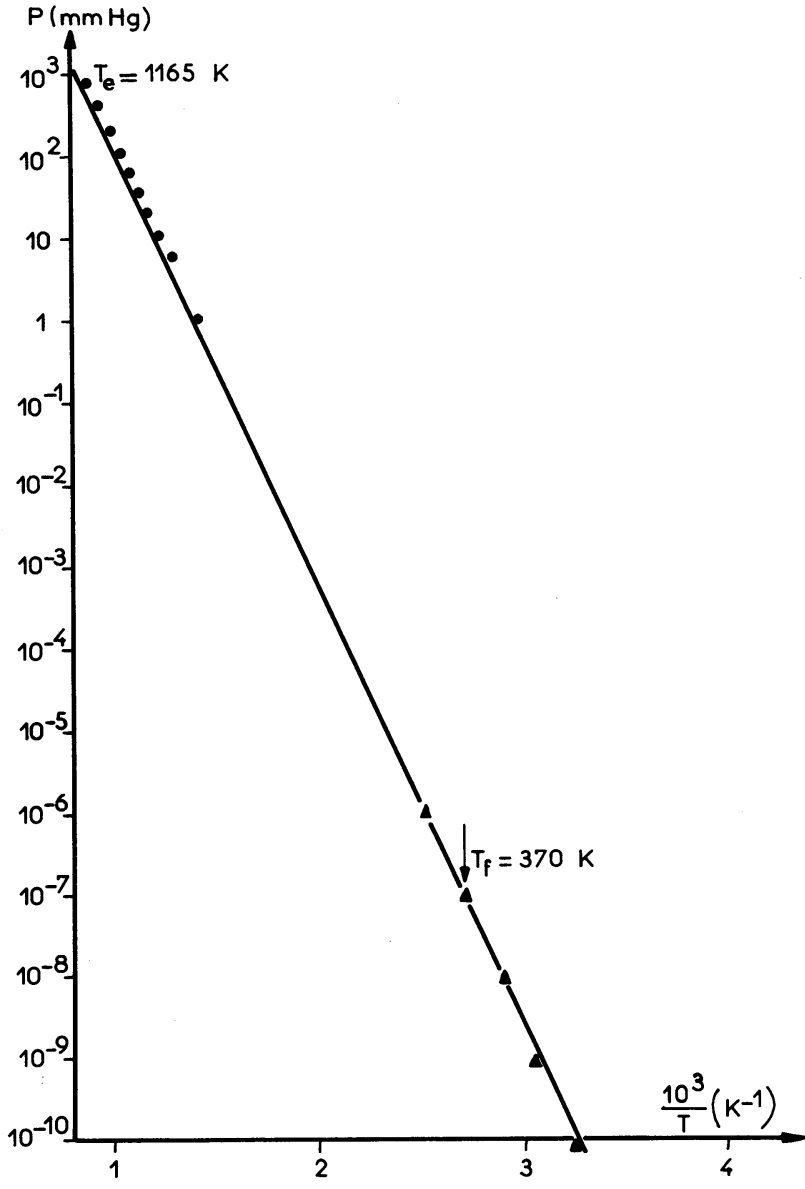

Fig. 8. - Variation de la pression de vapeur saturante du sodium en fonction de la température

$$
\log P=-5500 T^{-1}+7,551 \quad \text { [7] }
$$

- pression de vapeur saturante en équilibre avec $\mathrm{Na}$ liquide [9]; $\Delta$ pression de vapeur saturante en équilibre avec $\mathrm{Na}$ solide [8].

[Variation of the sodium saturating vapour pressure versus temperature

$$
\log P=-5500 T^{-1}+7.551 \quad \text { [7]; }
$$

- saturated vapour pressure balanced with liquid $\mathrm{Na}$ [9];

$\Delta$ saturating vapour pressure balanced with solid $\mathrm{Na}$ [8].]

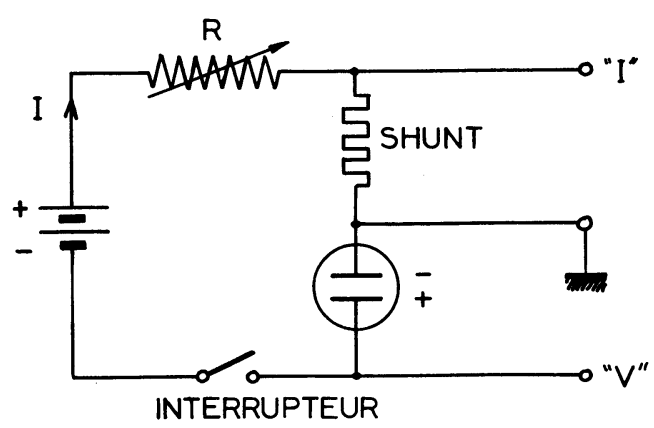

Fig. 9. - Etude du générateur en charge. Montage électrique. [Study of the generator on load. Electrical wiring.]

non représenté sur la figure permet d'imposer un courant positif (fonctionnement en générateur) ou négatif (fonctionnement en récepteur). La différence de potentiel $V$ et le courant $I$ sont enregistrés après stabilisation de la température (à $\pm 2,5^{\circ}$ ) de la source chaude $T_{1}$.

Les courbes de la figure 10 représentent l'évolution de la d.d.p. aux bornes du générateur en fonction du courant pour des températures comprises entre 270 et $530^{\circ} \mathrm{C}$. Il n'est pas possible de représenter cette évolution en fonction de la densité de courant, parce que les surfaces actives sont mal définies. Du côté de la source chaude, on peut admettre que le transfert de charges entre $\mathrm{Na}$ et $\mathrm{Na}^{+}$s'effectue sur toute la surface de l'alumine $\beta$ en contact avec le sodium liquide. Par contre, du côté de la source froide, la réduction des ions $\mathrm{Na}^{+}$en atomes métalliques ne peut se produire que dans les zones où il y a contact entre le fil de platine et l'alumine. Toute évaluation ne conduirait qu'à des valeurs très approximatives.

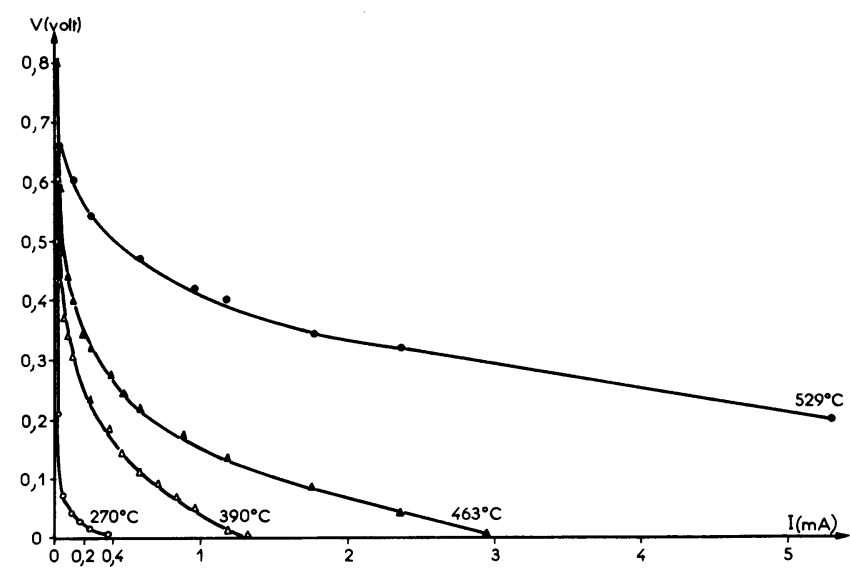

Fig. 10. - Essais en charge. Caractéristiques expérimentales intensité-potentiel pour différentes températures de source chaude.

[Device on load tests. Experimental curves intensity-potential for hot source different temperatures.]

L'examen de la figure 10 montre qu'à une température donnée, la d.d.p. aux bornes du générateur diminue lorsque le courant augmente. La décroissance est d'autant moins accentuée que la température est élevée. Considérons les étapes successives qui conduisent les atomes de sodium de la source chaude à la source froide. Les deux premières phases consistent en la réaction $\mathrm{Na} \rightarrow \mathrm{Na}^{+}+\mathrm{e}$ et la migration des ions $\mathrm{Na}^{+}$à travers l'électrolyte jusqu'au collecteur de platine. Ces deux processus s'effectuent avec une polarisation négligeable, comme le montrent les mesures de conductivité de l'alumine $\beta$ sur une cellule $(\mathrm{Na})_{\text {liq. }} / \mathrm{Al}_{2} \mathrm{O}_{3} \beta /(\mathrm{Na})_{\text {liq. }}$ [10], où interviennent les mêmes étapes. Il apparaît en effet que la résistance apparente est négligeable devant celles observées sur la figure 10. Il faut cependant préciser que dans le cas du générateur, la collection du courant, donc le transfert de charges, s'effectuent du côté de la source froide là où le fil de platine enroulé en spires non 
jointives est en contact avec le tube d'alumine. Les lignes de courant ne sont pas uniformément réparties à travers l'électrolyte et la résistance apparente associée à la migration est plus élevée que celle mesurée avec la cellule $(\mathrm{Na})_{\text {liq }} / \mathrm{Al}_{2} \mathrm{O}_{3} \beta /(\mathrm{Na})_{\text {liq. }}$.

L'étape suivante concerne la réaction de transfert de charges

$$
\mathrm{Na}^{+}+\mathrm{e} \rightarrow \mathrm{Na}
$$

au niveau du collecteur de platine. Il est difficile d'apprécier la polarisation introduite par cette réaction. En effet, la surface réactionnelle étant faible devant la surface totale de l'électrolyte, les densités de courant, donc les surtensions peuvent être importantes. Les constatations développées plus avant conduisent à penser que ce processus n'est pas limitatif dans le fonctionnement considéré.

La dernière phase correspond à la diffusion des atomes de sodium jusqu'à la source froide et à la condensation de la vapeur. La vitesse de ces processus étant limitée, le passage d'un courant s'accompagne dans le cas d'un fonctionnement en générateur d'une augmentation de la concentration en sodium (ou plus précisément de la pression) au niveau du collecteur et donc de l'apparition d'une surtension. Un calcul, développé à partir de l'équation de Fick [11], permet d'obtenir un ordre de grandeur de la polarisation associée à la diffusion. Dans le cas considéré, on obtient une polarisation de $0,6 \mathrm{~V}$ pour un courant de $1 \mathrm{~mA}$ et une température de $550 \mathrm{~K}$ [10]. Cette valeur ne constitue qu'une indication parce que de nombreux paramètres nécessaires au calcul sont seulement estimés. De plus, le transfert de masse s'effectue du collecteur à la source froide en présence d'un gradient thermique, et il est difficile d'admettre que la diffusion est seulement régie par la loi de Fick. Lorsqu'on considère un fonctionnement en récepteur, le même raisonnement conduit à prévoir une diminution de la concentration en sodium au niveau du collecteur. A une concentration nulle correspond le courant limite $I_{\mathrm{L}}$. Sur la figure 11, on constate effectivement une augmentation rapide de la différence de potentiel dans le cas d'un fonctionnement en récepteur $(I<0)$ suggérant l'existence de ce courant limite.

L'ensemble de ces observations montre que les étapes limitant les performances du générateur semblent être liées au transfert de matière du côté de la source froide. Nous envisageons actuellement de poursuivre cette étude en régime harmonique afin de préciser ce point.

4. Etude du rendement. -4.1 Rendement thÉoRIQUE. - Dans la conversion étudiée, le sodium liquide placé dans les conditions thermodynamiques de la source chaude $\left(p_{1}, T_{1}\right)$ se transforme en sodium liquide placé dans les conditions thermodynamiques de la source froide $\left(p_{2}, T_{2}\right)$. Afin de calculer le rendement théorique, il est nécessaire de considérer un cycle fermé et donc de prévoir les transformations per-

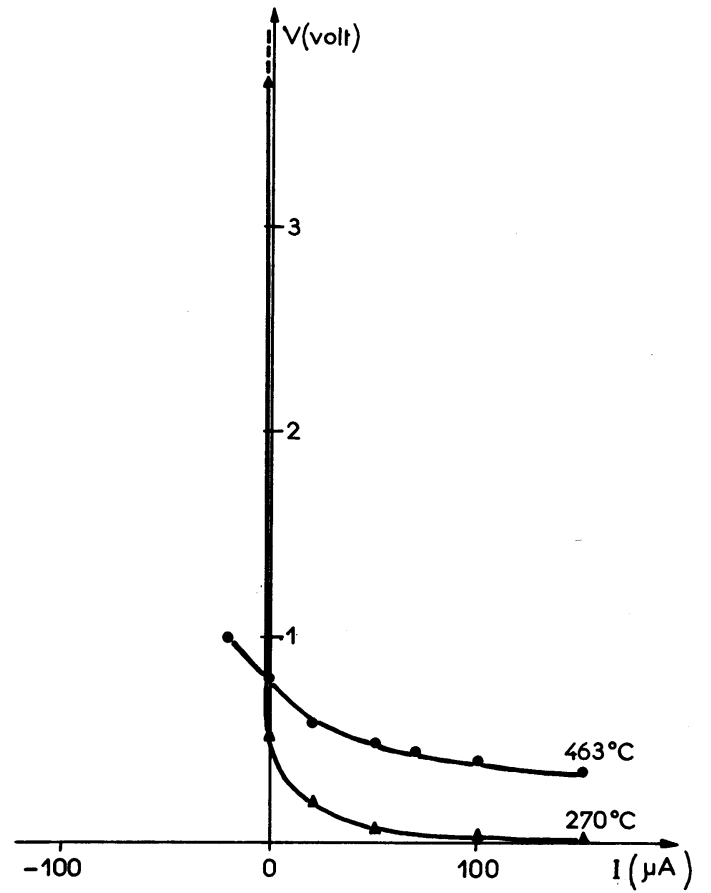

Fig. 11. - Essais en charge. Mise en évidence d'un courant limite lors du fonctionnement en récepteur.

[Device on load tests. Displaying the limit current when receptor is running.]

mettant de ramener le sodium de l'état $\left(p_{2}, T_{2}\right)$ à l'état $\left(p_{1}, T_{1}\right)$. Le cycle correspondant est tracé en coordonnées $(p, V)$ sur la figure 12 . La courbe en pointillé représente la courbe de saturation. Le maximum correspond au point critique. Afin de rendre la figure plus claire, l'échelle des abscisses est dilatée pour les faibles volumes (domaine du liquide).

Partons du point $A$. Celui-ci est associé à la vapeur de sodium située au-dessus de l'alumine $\beta$ (conditions

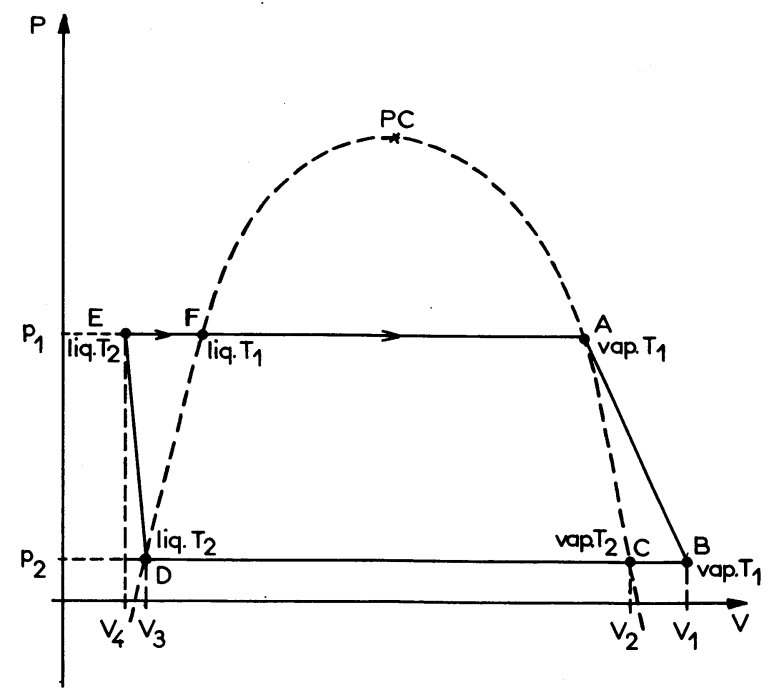

Fig. 12. - Etude du rendement. Diagramme $(P, V)$.

[Study on efficiency. $(P, V)$ diagram.] 
$\left.p_{1}, T_{1}\right)$. Nous appellerons première étape l'ensemble des processus suivants : oxydation du sodium en ions $\mathrm{Na}^{+}$, migration des ions $\mathrm{Na}^{+}$jusqu'à collecteur, réduction de ces ions en atomes de sodium. Ceux-ci sont alors dans l'état $\left(p_{2}, T_{1}\right)$. Nous supposerons que, du point de vue thermodynamique, cette étape peut être schématisée par une détente isotherme d'un gaz parfait. Dans ces conditions, la source chaude fournit une quantité de chaleur $Q_{1}$ reliée à $p_{1}$ et $p_{2}$ par la relation [12] :

$$
Q_{1}=R T_{1} \ln \frac{p_{1}}{p_{2}}
$$

Cette quantité de chaleur est transformée en énergie électrique lors de la réaction électrochimique et est cédée par le système au milieu extérieur. Si la transformation considérée s'accompagne de processus irréversibles, les pertes correspondantes sont récupérées par la source chaude. Il s'ensuit qu'à ce niveau le rendement partiel peut être pris égal à l'unité.

La seconde étape consiste en l'évolution sur le diagramme de $\mathrm{B}$ à $\mathrm{C}$. Ceci correspond au refroidissement de la vapeur de la température $T_{1}$ à la température $T_{2}$ (et donc à la diffusion de la vapeur de sodium jusqu'au niveau de la source froide). Cette transformation s'effectue en première approximation à pression constante. La chaleur cédée par le système est donnée par :

$$
Q_{2}=C_{p}\left(T_{2}-T_{1}\right)
$$

où $C_{p}$ est la chaleur molaire du gaz à pression constante.

De $\mathrm{C}$ à $\mathrm{D}$, la vapeur se condense à pression et température constante au niveau de la source froide. La quantité de chaleur mise en jeu est :

$$
Q_{3}=-L_{2}
$$

où $L_{2}$ est la chaleur latente de vaporisation du sodium à la température $T_{2}$. Celle-ci est reliée à la température par [13] :

$$
L=25,95-2,34 \times 10^{-3} T
$$

où $T$ est exprimé en $\mathrm{K}$ et $L$ en kcal.mole ${ }^{-1}$.

Le point $\mathrm{D}$ du diagramme correspond à l'état final dans lequel se trouve le sodium lors du fonctionnement en charge, précédemment décrit.

Envisageons alors une compression isotherme correspondant à l'évolution de $\mathrm{D}$ à $\mathrm{E}$. La compressibilité d'un liquide étant beaucoup plus faible que celle d'un gaz, on peut admettre que la quantité de chaleur échangée avec l'extérieur est nulle [14] :

$$
Q_{3}^{\prime}=R T_{2} \ln \frac{V_{4}}{V_{3}} \approx 0
$$

Cette compression isotherme doit être suivie d'un échauffement du liquide effectué à pression constante $($ de $E$ à $F$ ). La chaleur fournie au système est égale à :

$$
Q_{4}=C_{p}^{\prime}\left(T_{1}-T_{2}\right)
$$

où $C_{p}^{\prime}$ est la chaleur molaire du liquide à pression constante.

Enfin, l'étape de $\mathrm{F}$ et à $\mathrm{A}$ correspond à la vaporisation du liquide à pression et température constantes. La quantité de chaleur mise en jeu est :

$$
Q_{5}=L_{1}
$$

où $L_{1}$ est la chaleur de vaporisation du sodium liquide à la température $T_{1}$.

Le long du cycle fermé ABCDEFA, la variation de l'énergie interne du sodium est nulle et la somme des travaux et des quantités de chaleur mis en jeu le long du cycle est égale à 0 , soit :

$$
W+Q_{1}+Q_{2}+Q_{3}+Q_{3}^{\prime}+Q_{4}+Q_{5}=0
$$

$Q_{2}, Q_{3}$ et $Q_{3}^{\prime}$ sont des quantités de chaleur cédées au milieu extérieur. Le rendement est défini comme le rapport du travail fourni par le système et des quantités de chaleur fournies au système. En fait, le seul travail récupéré est celui fourni par le générateur sous forme électrique. Ce travail $W_{\mathrm{G}}$ est égal à la quantité de chaleur $Q_{1}$ fournie par la source chaude lors du trajet $\mathrm{AB}$ (détente isotherme), soit :

$$
W_{\mathrm{G}}=-Q_{1} \text {. }
$$

Les quantités de chaleur cédées au sodium sont $Q_{1}, Q_{4}$ et $Q_{5}$ correspondant respectivement aux trajets $\mathrm{AB}, \mathrm{EF}$ et FA. Le rendement théorique s'écrit dans ces conditions :

$$
\eta_{\mathrm{G}}=\frac{Q_{1}}{Q_{1}+Q_{4}+Q_{5}} .
$$

Précisons que, si des dissipations se produisent lors de la réaction électrochimique, la quantité de chaleur correspondante $q$ est récupérée par la source chaude. Le rendement devient alors :

$$
\eta_{\mathrm{G}}=\frac{Q_{1}-q}{Q_{1}+Q_{4}+Q_{5}-q} .
$$

Cette expression montre que le rendement est moins affecté par les processus irréversibles que celui associé aux convertisseurs électrochimiques classiques. En effet dans ce cas, les dissipations entraînent un transfert de chaleur du générateur au milieu extérieur sans qu'il soit possible de récupérer cette énergie.

En remplaçant $Q_{1}, Q_{4}$ et $Q_{5}$ par leur valeur en fonction de la pression et de la température, la relation précédente s'écrit :

$$
\eta_{\mathrm{G}}=\frac{R T_{1} \ln \frac{p_{1}}{p_{2}}}{R T_{1} \ln \frac{p_{1}}{p_{2}}+C_{p}^{\prime}\left(T_{1}-T_{2}\right)+L_{1}} .
$$


On sait que dans cette expression $p_{1}$ et $p_{2}$ représentent les pressions de vapeur saturante du sodium respectivement à $T_{1}$ et $T_{2}$. La variation de $p$ en fonction de $T$ est donnée par [15] :

$$
\frac{\mathrm{d} \ln p}{\mathrm{~d} T}=\frac{L}{R T^{2}}
$$

où $L$ est la chaleur latente de vaporisation à la température $T$. Dans le cas présent, l'équation (3) montre que $L$ est pratiquement constant dans le domaine de température concerné $\left(L_{1} \approx L_{2} \approx L\right)$. Dans ces conditions, on a :

$$
\ln \frac{p_{1}}{p_{2}}=\frac{L}{R}\left(\frac{1}{T_{2}}-\frac{1}{T_{1}}\right)
$$

et, le rendement $\eta_{\mathrm{G}}$ est donné par :

$$
\eta_{\mathrm{G}}=\frac{L\left(\frac{T_{1}}{T_{2}}-1\right)}{L \frac{T_{1}}{T_{2}}+C_{p}^{\prime}\left(T_{1}-T_{2}\right)}
$$

ou en faisant apparaître le rendement de Carnot $\eta_{\mathrm{C}}$ :

$$
\eta_{\mathrm{G}}=\frac{\eta_{\mathrm{C}}}{1+\frac{C_{p}^{\prime}}{L} \frac{T_{2}}{T_{1}}\left(T_{1}-T_{2}\right)} .
$$

Le dénominateur étant positif, le rendement théorique est évidemment inférieur au rendement de Carnot. Nous avons porté sur le tableau ci-dessous les valeurs calculées pour différentes températures de source chaude et une température de source froide égale à $370 \mathrm{~K}\left(\approx 100^{\circ} \mathrm{C}\right)$.

$\begin{array}{cccc}T_{1}(\mathrm{~K}) & T_{2}(\mathrm{~K}) & \eta_{\mathrm{C}} & \eta_{\mathrm{G}} \\ \overline{543} & - & - & - \\ 664 & 370 & 0,31 & 0,30 \\ 736 & 370 & 0,44 & 0,42 \\ 802 & 370 & 0,53 & 0,50\end{array}$

Les températures de source chaude choisies correspondent à celles fixées lors des essais. On constate que le rendement théorique du générateur est peu inférieur au rendement de Carnot et qu'il est relativement élevé. Le rendement réel du convertisseur est évidemment plus faible à cause notamment des dissipations au niveau de la conversion électrochimique, et des transferts de chaleur de la source chaude à la source froide le long des parois de la cellule.

4.2 ReNDEMENT RÉEL DU CONVERTISSEUR. - Dans le générateur étudié, le sodium décrit un cycle ouvert. Il n'est donc pas possible de déterminer expérimentalement le rendement de la conversion. On peut néanmoins, par la mesure ou le calcul, estimer certains facteurs qui provoquent des pertes autres que celles définies dans le paragraphe précédent.
Un de ces facteurs résulte de l'existence de processus irréversibles qui sont également à l'origine de la décroissance de la d.d.p. aux bornes du générateur en fonction du courant débité. Afin d'apprécier leur influence sur le rendement, nous allons préciser la nature de ces processus.

En premier lieu, le passage du courant s'accompagne d'une polarisation associée aux transferts de charges lors des réactions électrochimiques et à la migration des ions $\mathrm{Na}^{+}$à travers l'alumine $\beta$. Il en résulte des dissipations de chaleur $q$ qui affectent l'énergie électrique récupérée $W_{\mathrm{G}}$. Celle-ci s'écrit avec les notations définies au paragraphe 4.1 :

$$
W_{\mathrm{G}}=-\left(Q_{1}-q\right) \text {. }
$$

On notera que l'énergie $q$ est dissipée dans une zone dont la température peut être considérée comme celle de la source chaude. Ainsi, la quantité de chaleur que doit fournir la source chaude est égale à $Q_{1}-q$. Dans ces conditions, le rendement devient

$$
\eta=\frac{Q_{1}-q}{Q_{1}-q+Q_{4}+Q_{5}} .
$$

Le transport des atomes de sodium formés jusqu'à la source froide apparaît comme une seconde étape limitative. La surtension associée à ce processus rend compte de l'augmentation de la pression partielle du sodium au niveau du collecteur de platine et donc d'une diminution du gradient d'activité à travers l'électrolyte. Dans ces conditions, l'état final correspondant à la première étape du cycle thermodynamique décrit par le sodium (cf. paragraphe précédent) n'est pas défini par $\left(p_{2}, T_{1}\right)$ mais par $\left(p_{2}^{\prime}, T_{1}\right)$ ou $p_{2}^{\prime}$, compris entre $p_{1}$ et $p_{2}$, a une valeur d'autant plus élevée que le courant est important. La quantité de chaleur fournie par la source chaude et l'énergie électrique récupérée sont alors respectivement $Q_{1}^{\prime}$ et $W_{\mathrm{G}}^{\prime}$ entre lesquelles l'égalité suivante reste vérifiée :

$$
W_{\mathrm{G}}^{\prime}=-Q_{1}^{\prime} .
$$

L'expression du rendement s'écrit finalement :

$$
\eta=\frac{Q_{1}^{\prime}-q}{Q_{1}^{\prime}-q+Q_{4}+Q_{5}} .
$$

Les quantités $Q_{4}$ et $Q_{5}$ ne sont pas affectées par les processus irréversibles qui viennent d'être décrits. Dans l'équation (5) $Q_{1}^{\prime}-q$ représente l'énergie électrique effectivement récupérée, tandis que dans (4), le terme $Q_{1}$ correspond à l'énergie électrique susceptible d'être recueillie en l'absence de polarisation. Pour un point de fonctionnement donné, la valeur de $Q_{1}^{\prime}-q$ est donc reliée à celle de $Q_{1}$ par :

$$
Q_{1}^{\prime}-q=\frac{V}{E} Q_{1}
$$

où $V$ est la d.d.p. aux bornes de la cellule et $E$ la f.é.m. L'examen des courbes de la figure 10 permet de 
prévoir une diminution du rendement lorsque le courant augmente ou que la température décroît. Nous avons calculé la valeur du rendement dans le cas où la température de la source chaude est $T_{1}=802 \mathrm{~K}$ et pour des courants de 3 et $5 \mathrm{~mA}$. On trouve pour :

$$
\begin{array}{lll} 
& I=3 \mathrm{~mA} & \eta=19 \% \\
\text { et pour } & I=5 \mathrm{~mA} & \eta=14 \% .
\end{array}
$$

Rappelons que le rendement théorique est égal à $50 \%$. Ces valeurs numériques montrent qu'il est important de diminuer la polarisation. Ceci peut être obtenu en augmentant $T_{1}$ et en concevant une cellule dont la structure favorise les phénomènes de diffusion.

Il se produit, par ailleurs, des échanges de chaleur entre la source chaude et la source froide du fait de l'existence d'une liaison matérielle entre ces deux zones. Le phénomène mis en cause est principalement la conduction à travers les parois du tube enveloppe. La quantité de chaleur transférée par unité de temps est proportionnelle au gradient thermique le long du tube considéré, à la section et à la conductivité thermique du matériau (du verre Pyrex dans le cas présent). Toutes choses étant égales par ailleurs, les pertes sont d'autant plus importantes que la température de la source chaude est élevée. Cependant, l'élévation de température permet d'obtenir des courants plus importants et donc de diminuer le temps nécessaire à la transformation d'une quantité donnée de sodium, une mole par exemple. (Nous rappelons que les quantités de chaleur intervenant dans l'expression du rendement sont des grandeurs molaires.) Il en résulte que, malgré l'augmentation de la puissance transférée par conduction de la source chaude à la source froide, la quantité de chaleur correspondante $Q_{\mathrm{c}}$, ramenée à une mole de sodium, diminue - ce qui illustre l'action indirecte des phénomènes de polarisation sur $Q_{\mathrm{c}}$ - . Dans ces conditions, le rendement devient :

$$
\eta=\frac{Q_{1}^{\prime}-q}{Q_{1}^{\prime}-q+Q_{4}+Q_{5}+Q_{\mathrm{c}}} .
$$

Pour une température de la source chaude de $802 \mathrm{~K}$ et pour un courant de $5 \mathrm{~mA}$, ceci conduit à un rendement de $6 \%$. L'amélioration du rendement nécessite de mettre au point une structure qui, compte tenu des contraintes technologiques, minimise les pertes par conduction.

5. Conclusion. - L'analyse des courbes décrivant le fonctionnement en charge du générateur suggère que les performances dépendent en grande partie des processus qui se développent entre le collecteur et la source froide, à savoir :

- la diffusion des atomes formés jusqu'à la source froide,

- la condensation au niveau de la source froide.

Des mesures en régime harmonique devraient permettre de préciser l'influence de ces deux processus sur la polarisation du générateur.

Par ailleurs, l'existence d'un gradient thermique du collecteur à la source froide rend possible, à chaque température intermédiaire, l'établissement de multiples équilibres condensation-vaporisation auxquels doivent être associées différentes cinétiques. Aussi est-il difficile de caractériser par un seul paramètre l'ensemble de ces processus. La diffusion est accélérée lorsque la température augmente. Par contre, les phénomènes de condensation seront d'autant moins limitatifs que la température de la source froide sera basse. Ceci suggère de réaliser une cellule comprenant une zone présentant un fort gradient de température. Mais on risque alors de favoriser les transferts de chaleur par conduction de la source chaude à la source froide. Aussi nous semble-t-il que l'étude des phénomènes de condensation, et de leur influence relative, devrait conduire à des solutions technologiques mieux adaptées que celles mises en œuvre dans le présent travail.

L'ensemble des expériences montre que l'élévation de la température de la source chaude s'accompagne d'une augmentation de la force électromotrice et d'une diminution de la chute de tension en charge. L'utilisation de tels générateurs se conçoit avec une température de source chaude proche de celle d'ébullition du sodium $\left(892^{\circ} \mathrm{C}\right)$, sous réserve d'utiliser des matériaux résistant aux températures. Les performances pourraient être alors du même ordre de grandeur que celles obtenues avec des générateurs électrochimiques monothermes $\left(\sim 100 \mathrm{~mW} . \mathrm{cm}^{-2}\right)$. Le rendement aurait une valeur sensiblement supérieure à celles obtenues dans le cadre de la présente expérience, et l'application de ces générateurs au domaine de la conversion solaire pourrait être envisagée.

Le dernier point concerne le vieillissement de l'électrolyte. En effet, la conductivité relativement élevée de l'alumine $\beta$ est due à l'existence de plans entre lesquels s'effectue la migration des ions $\mathrm{Na}^{+}$. L'introduction d'ions métalliques étrangers $\left(\mathrm{K}^{+}\right.$en particulier) a pour effet de réduire la mobilité des ions $\mathrm{Na}^{+}$. La durée de fonctionnement sera donc fonction de la pureté du sodium. Les recherches actuelles sur les électrolytes solides devraient permettre de se libérer de cette contrainte et, à moyen terme, d'envisager l'utilisation d'autres métaux. 


\section{Bibliographie}

[1] Whittingham, M. S., Huggins, R. A., Proceedings of the 5th Materials Research Symposium, p. 139, National Bureau of Standars Special Publication 364, Solid State chemistry (1972).

[2] Weiner, S. A., Research on Electrodes and Electrolytes for the Ford Sodium-Sulfur Battery, Contract WSFC 805 National Science Foundation, U.S.A. (1975).

[3] BodKRIS, J. O'M., REDDY, A. K. N., Modern Electrochemistry 2 (Plenum Press, N. Y.) 1972, p. 901.

[4] Broussaud, D., Lazennec, Y., Brevet ANVAR No 74 31217, France (1974).

[5] Lazennec, Y., Etude de l'évolution d'un électrolyte en alumine $\beta$ pour accumulateur sodium-soufre, Rapport de fin de Contrat DGRST n $^{\circ}$ 73-7-1087 (1974).

[6] Kummer, J. T., Weber, N., United State Patent 3458-356, U.S.A. (1969).
[7] Pascal, P., Nouveau traité de Chimie Minérale 6 (Masson éditeur) 1934 , p. 380.

[8] Maissel, L. I., Glang, R., Handbook of thin film technology (Mac Graw Hill Ed.) 1970, p. 1-16.

[9] GRAY, D. E., American Institute of Physics Handbook (Mc Graw Hill Ed.) 1963, p. 4-226.

[10] Loge, B., Thèse de Docteur-Ingénieur, Université Paris 6 (1979).

[11] Jost, J. W., Diffusion in solids, liquids, gases (Academic Press, N.Y.) 1970 , p. 3.

[12] Rocard, Y., Thermodynamique (Masson editeurs) 1952, p. 9.

[13] Landendurg, Minskovski, Z. Phys. 6 (1921) 162.

[14] BRuhat, G., Thermodynamique (Masson éditeur) 1962, p. 150.

[15] BRuhat, G., ibid., p. 280. 